\section{The effects of core training given to female soccer players on different vertical jumping methods}

\section{Kadın futbolculara uygulanan kor kuvvet antrenmanlarının farklı dikey sıçrama yöntemlerine etkisi}

\author{
Mehmet Göktepe ${ }^{1}$ \\ Meral Miyaç Göktepe ${ }^{2}$ \\ Ferhat Güder ${ }^{3}$ \\ Mehmet Günay ${ }^{4}$
}

Abstract

This study aims to determine the effects of core training given to female soccer players on their methods of different vertical jumping. 16 female certified soccer players playing in the female soccer team of Bartin University with average age of $21.07 \pm 3.56$ years, average height of $163.00 \pm 7.18 \mathrm{~cm}$ and with weight of $56.60 \pm 7.15 \mathrm{~kg}$ were included in the study on the basis of volunteering. Prior to the tests, all the female soccer players completed a survey form used in determining their medical status and filled in a form to confirm that they participated in the study voluntarily. The core training programme was applied for 8 weeks two times a week regularly. The pre-test and post-test measurements were made and then subjects' height and weight were measured, they were asked to run for 10 minutes for warm-up and the activity was ended by making jumping measurement. The research was performed in the physiology laboratory of Bartun University School of Physical Education and Sport (BESYO). Statistical evaluation was made by using SPSS 22.0 programme. The values

\section{Özet}

$\mathrm{Bu}$ araştırmanın amacı, kadın futbolculara uygulanan kor kuvvet antrenmanlarının farklı dikey sıçrama yöntemlerine etkisinin belirlenmesidir. Çalışmaya Bartın Üniversitesi Kadın Futbol Takımında lisansli, yaş ortalamalar1 $21,07 \pm 3,56 \mathrm{y} 1$, boy ortalamalar1 $163,00 \pm 7,18 \quad(\mathrm{~cm})$ ve vücut ağ1rlikları $56,60 \pm 7,15(\mathrm{~kg})$ olan gönüllü 16 kadin futbolcu dahil edilmiştir. Testlerden önce araştırmaya katılan kadın futbolcular sağlık durumlarını belirtir sağlık anketini ve gönüllü katılım formunu doldurup imzalamişlardir. Kor antrenman program1 8 hafta boyunca, haftada 2 gün düzenli olarak uygulanmıştır. Çalışmaya katılan kadın futbolculara sırasıyla; boy ve vücut ağ1rlı̆̆1 ölçümü yapıldı. 10 dakika 1sınma koşusu yaptırıldıktan sonra, sıçrama ölçümleri yapılarak çalışma sonlandırıldı. Bu araştırma Bartın Üniversitesi BESYO Fizyoloji Laboratuvarında yapılmıştır. İstatistiksel değerlendirme SPSS 22.0 programı kullanilarak yapıld1. Ön test ve son testten elde edilen değerler, Shapiro-Wilk testi ile normallik dağılımına bakıldıktan sonra Wilcoxon İşaretli Sıralar testi yardımı ile

\footnotetext{
${ }^{1}$ Dr. Öğr. Üyesi, Balıkesir Üniversitesi, Beden Eğitimi ve Spor Yüksekokulu, mgoktepe06@gmail.com

${ }^{2}$ Doktora Öğrencisi, Gazi Üniversitesi, Sağlık Bilimleri Enstitüsü, meral.miyac@,hotmail.com

3 Ögr. Gör., Bartın Üniversitesi, Beden Eğitimi ve Spor Yüksekokulu, ferhatguder1981@gmail.com

${ }_{4}^{4}$ Prof. Dr., Gazi Üniversitesi, Spor Bilimleri Fakültesi, mgunay@gazi.edu.tr
} 
Göktepe, M., Göktepe, M. M., Güder, F., \& Günay, M. (2019). Kadın futbolculara uygulanan kor kuvvet antrenmanlarının farklı dikey sıçrama yöntemlerine etkisi. Journal of Human Sciences, 16(3), 791-798. doi:10.14687/jhs.v16i3.5637

obtained were compared after checking normality distribution through Shapiro-Wilk test with the help of Wilcoxon signed rank test. This study, we aimed to determine the effect of core strength training on different vertical jump methods applied to female soccer players. It has been observed that it has a favorable effect on counter movement jumping (CMJ) and static jumping $(\mathrm{SJ})$ values $(\mathrm{p}<0.05)$. It was found that there was no statistically significant effect on drop jumping (DJ) values ( $p>0.05)$. As a result; It can be said that the core strength training practiced in female soccer players developed counter movement jumping_and static jumping characteristic of vertical jumping methods and did not affect the drop jumpingfeature. In line with these results, it is recommended to take into account the core training programs because the strength training that will be applied in female soccer players can improve the counter movement jumping and static jumping feature.

Keywords: Soccer; core training; vertical jump; static jump; counter movement jump; drop jump.

(Extended English summary is at the end of this document)

\begin{abstract}
istatistiksel
olarak karşılaştırılmıştır. Çalışmamızın bulgularına göre; kadın futbolculara uygulanan kor kuvvet antrenmanlarının farklı dikey sıçrama yöntemlerine etkisinin belirlenmesi amaciyla yapmış olduğumuz çalışmamızda uygulanan kor kuvvet antrenmanlarının dikey sıçrama yöntemlerinden; yaylanarak sıçrama (YS) ve statik sıçrama (SS) değerlerine olumlu düzeyde etki ettiği gözlemlenmiştir $(\mathrm{p}<0.05)$. Düsserek siçrama (DS) değerlerine ise istatistiksel olarak etkisinin olmadığ1 tespit edilmiştir ( $\mathrm{p}>0.05)$. Sonuç olarak; kadın futbolcularda uygulanan kor kuvvet antrenmanlarının dikey sıçrama yöntemlerinden statik sıçrama ve yaylanarak sıçrama özelliğini geliştirdiği, düşerek sıçrama özelliğine ise etki etmediği söylenebilir. Bu sonuçlar doğrultusunda kadın futbolcularda uygulanacak kor kuvvet antrenmanlarının statik siçrama ve yaylanarak sıçrama özelliğine olumlu yönde etkisi olabileceğinden antrenman programlarında göz önünde bulundurulması önerilebilir.
\end{abstract}

Anahtar Kelimeler: Futbol; kor kuvvet; dikey sıçrama; statik sıçrama; yaylanarak sıçrama; düşerek sıçrama.

\section{Giriş}

Futbolda kısa mesafeli sprintler, yön değiştirmeler, ani duruşlar, kafa vuruşu, sıçrama ve topa vurma gibi kısa sürede ve yüksek şiddette meydana gelen anaerobik enerji ile ilgili hareketler sıklıkla meydana gelmektedir (Günay ve Yüce, 2008; Yildiz, 2018). Dikey sıçrama, atlama ve sıçrama hareketlerini içeren aktivitelerde alt ekstremitenin sergilediği patlayıcı kuvvet yeteneğidir (Günay ve ark., 2006; Tamer, 2000). Sportif performans belirli bir spor motorik düzeyin biçimlenme derecesidir. Kompleks yapısından dolayı spesifik faktörleri içermektedir ve performans gelişimi için yapılan antrenmanlarında çok yönlü olması zorunluluğu vardır. Performansı belirleyen faktörlerin uyumlu gelişimi ile bireysel maksimum başarıya ulaşılmaktadır (Günay ve Yüce, 2008). Dikey sıçrama rekabet gücü en yüksek sporlarda kullanılan temel bir beceridir (Baljinder ve ark., 2014). Antrenman rejimlerinin birçoğunda amaç ilgili sporda atletin performansını artırmak için dikey sıçrama yeteneğini en üst düzeye çıkarmaya çalışmaktır (Baljinder ve ark., 2014; Reiser ve ark., 2006). Siçrama ve çabukluk gerektiren sporlarda, kassal kuvvet ve güce gereksinim vardır (Baljinder ve ark., 2014; Semler, 2011). Futbolcuların kuvvet gelişiminde en çok tercih edilen yöntemlerden biri de kor antrenmandır (Riewald, 2003). Kor kavramı sternum ve dizler arasında kalan, abdominal bölge, bel ve kalçaları kapsayan kısım olarak ele alınmaktadır (Santana, 2005). Bireyin kendi vücut ağırlı̆̆ ile gerçekleştirdiği, omurgayı dengede tutmakta olan derin kasların ve lumbo pelvik bölgede yer alan kaslarının kuvvetlendirilmesini amaçlayan egzersiz programına kor antrenman denmektedir (Atan ve ark., 2013). Kor antrenman bölgesel ve yüzeysel kasların kuvvet ve kondisyonu üzerinde durur (Clark, 2001). Kor antrenman son y1llarda büyük bir ilgi görmekte ve antrenman planlarının temel bir parçası haline gelmeye başlamıştır (Riewald, 2003). Literatüre 
Göktepe, M., Göktepe, M. M., Güder, F., \& Günay, M. (2019). Kadın futbolculara uygulanan kor kuvvet antrenmanlarının farklı dikey sıçrama yöntemlerine etkisi. Journal of Human Sciences, 16(3), 791-798. doi:10.14687/jhs.v16i3.5637

baktığımızda kor kuvvet antrenmanlarının sıçrama kuvvetine etki ettiği çalışmalara çok sayıda rastlanmaktadır. Fakat dikey sıçrama yöntemleri içerisinde statik sıçrama, yaylanarak sıçrama ve düşerek sıçramaya ayrı ayıı nasıl etki ettiğiyle ilgili az sayıda çalışmaya rastlanmaktadır. Ayrıca kadın futbolcular üzerine yapılmış çalışmalar, erkek futbolcularla yapılmış çalışmalardan daha azdır. Bu nedenle çalışma bulgularının literatüre katkı sağlaması açısından önemli olduğu söylenebilir. $\mathrm{Bu}$ bağlamda çalışmamızda kadın futbolculara uygulanan kor kuvvet antrenmanlarının farklı dikey sıçrama yöntemlerine etkisinin belirlenmesi amaçlanmaktadır.

\section{Yöntem}

\subsection{Araştırma Grubu}

Araştırma grubunu, Bartın Üniversitesi Kadın Futbol Takımında lisanslı, yaş ortalamaları

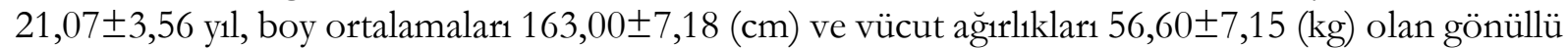
14 kadın futbolcu oluşturmaktadır.

\subsection{Veri Toplama Araçları}

Boy uzunluğu ve Vücut Ağgrllğ Ölçümleri: Deneklerin boy uzunlukları hassaslık derecesi $0.01 \mathrm{~m}$ olan (SECA, Almanya) boy ölçer ile vücut ağırlığ ölçümleri ise hassaslık derecesi $0.1 \mathrm{~kg}$ olan elektronik baskülle (SECA, Almanya) ölçülmüştür.

Vücut Kitle Indeksi (VKI): VKİ, olguların vücut ağırlıklarının kg değerinin, boy uzunluğu metre ölçümünün karesine bölünmesi ile $\left(\mathrm{kg} / \mathrm{m}^{2}\right)$ hesaplanmıştır (Moran ve McGlynn, 1997; Norris ve ark., 2005; Taylor ve ark., 1998).

Dikey Sıcrama Testleri: Sporcuların dikey sıçrama özellikleri; Statik, Yaylanarak ve Düşerek Siçrama yöntemleri Powertimer PC 1.9.5 Version Newtest cihazı ve cihaza bağlı "mat" ve bilgisayar programı kullanılarak ölçüldü. Sporcular tüm sıçrama testlerini 3' er kez yaptı. Tüm sıçrama değerlerinin ortalamaları alınarak kayıt edildi. Sıçrama değerleri; Sıçrama yüksekliği (cm) (SY), Siçrama gücü (watt) (SG) olarak kaydedildi.

Statik Sıçrama (SS): Sporcular eller belde çift ayakla "mat"1n üzerinde dizler $90^{\circ}$ squatta olacak şekilde dururlarken siçrayabildikleri kadar yukarı sıçradılar.

Yaylanarak Sıçrama (YS): Sporcular eller belde çift ayakla matın üzerinde dizler $90^{\circ}$ squatta hızlı bir şekilde inerek sıçrayabildikleri kadar yukarı sıçradılar

Düserek Sıçama (DS): Sporcular $40 \mathrm{~cm}$ yükseklikteki bir basamağın üzerinde eller belde çift ayakla yerde duran matın üzerine düştüler ve ayakları mata temas eder etmez yarım squatla sıçrayabildikleri kadar yukarı sıçradılar (Çon ve ark., 2012).

\subsection{Verilerin Toplanmas1}

Bütün sporcular testlerden önce sağlık durumlarının belirlenmesinde kullanılan sağlık anketini ve çalışmaya gönüllü katıldıklarını belirten formu doldurup imzalamışlardır. Çalışmaya katılmayı kabul eden her adaya öncelikle çalışmanın içeriği tüm ayrıntıları ile anlatılmıştır. Ölçümler yapılmadan önce bütün katılımcılar ölçümden önceki günde ağır egzersiz yapmamaları ve alkol tüketmemeleri konusunda uyarılmıştır. Deneklere sırasıyla; 1) Boy ve kilo ölçümü yapıldı. 2) 10 dakika 1sınma koşusu yaptırıldı. 3) Sıçrama ölçümleri yapılarak çalışma sonlandırıldı. Bu araştırma Bartın Üniversitesi BESYO Fizyoloji Laboratuvarında yapılmışıır.

\subsection{Verilerin Analizi}

İstatistiksel değerlendirme SPSS 22.0 (SPSS Inc., Chicago, IL, USA) programı kullanılarak yapıldı. Ön test ve son testten elde edilen değerler, Shapiro-Wilk testi ile normallik dağılımına bakıldıktan sonra Wilcoxon İşaretli Sıralar testi yardımı ile istatistiksel olarak karşılaştırılmıştır. Bütün istatistiksel yöntemler için yanılma düzeyi $(\alpha) 0,05$ olarak kabul edilmiştir. 
Göktepe, M., Göktepe, M. M., Güder, F., \& Günay, M. (2019). Kadın futbolculara uygulanan kor kuvvet antrenmanlarının farklı dikey sıçrama yöntemlerine etkisi. Journal of Human Sciences, 16(3), 791-798. doi:10.14687/jhs.v16i3.5637

\section{Bulgular}

Tablo 1: Çalışmaya katılan kadın futbolcuların fiziksel özellikleri

\begin{tabular}{llllll}
\hline $\mathrm{N}$ & Yaş (y1l) & Boy Uzunluğu (cm) & Vücut Ağırlı̆g $(\mathrm{kg})$ & $\mathrm{VKI}(\mathrm{kg} / \mathrm{m} 2)$ & Spor Yaşı (y1l) \\
\hline 14 & $21,07 \pm 3,56$ & $163,00 \pm 7,18$ & $56,60 \pm 7,15$ & $21,32 \pm 2,52$ & $5,21 \pm 3,31$ \\
\hline
\end{tabular}

Tablo 1'de araştırmaya katılan kadın futbolcuların yaş, boy, vücut ağrllı̆̆, VKİ ve spor yaşlanı verilmiştir. Sporcuların yaşları $21,07 \pm 3,56$ yll, boy uzunlukları $163,00 \pm 7,18 \mathrm{~cm}$, vücut ağırlıkları

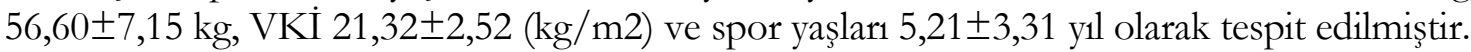

Tablo 2. Araştırmaya katılan kadın futbolcuların ön test ve son test parametreleri arasındaki farkın anlamlılığını test etmek için yapılan wilcoxon işaretli sıra testi sonuçları

\begin{tabular}{|c|c|c|c|c|c|c|c|}
\hline \multicolumn{4}{|c|}{ Parametreler } & $\mathrm{n}$ & Ortalama & SS & $\mathrm{P}$ \\
\hline \multirow{2}{*}{\multicolumn{3}{|c|}{ Boy $(\mathrm{cm})$}} & Ön test & 14 & 163,00 & 7,18 & \multirow{2}{*}{ - } \\
\hline & & & Son test & 14 & 163,00 & 7,18 & \\
\hline \multirow{2}{*}{\multicolumn{3}{|c|}{ Vücut Ağırlı̆̆1 (kg) }} & Ön test & 14 & 56,71 & 6,99 & \multirow{2}{*}{0,180} \\
\hline & & & Son test & 14 & 56,50 & 7,32 & \\
\hline \multirow{2}{*}{\multicolumn{3}{|c|}{ VKİ (kg/m2) }} & Ön test & 14 & 21,37 & 2,47 & \multirow{2}{*}{0,180} \\
\hline & & & Son test & 14 & 21,28 & 2,58 & \\
\hline \multirow{12}{*}{ 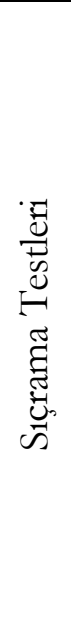 } & \multirow{4}{*}{ SS } & \multirow{2}{*}{$\mathrm{SY}(\mathrm{cm})$} & Ön test & 14 & 29,09 & 4,57 & \multirow{2}{*}{$0,030^{(*)}$} \\
\hline & & & Son test & 14 & 31,14 & 5,51 & \\
\hline & & \multirow{2}{*}{ SG (watt) } & Ön test & 14 & 2351,00 & 487,13 & \multirow{2}{*}{$0,030^{(*)}$} \\
\hline & & & Son test & 14 & 2475,36 & 510,06 & \\
\hline & \multirow{4}{*}{ YS } & \multirow{2}{*}{$\mathrm{SY}(\mathrm{cm})$} & Ön test & 14 & 36,13 & 14,62 & \multirow{2}{*}{$0,033^{(*)}$} \\
\hline & & & Son test & 14 & 39,87 & 19,48 & \\
\hline & & \multirow{2}{*}{ SG (watt) } & Ön test & 14 & 2771,07 & 882,07 & \multirow{2}{*}{0,064} \\
\hline & & & Son test & 14 & 2862,71 & 1021,08 & \\
\hline & \multirow{4}{*}{ DS } & \multirow{2}{*}{$\mathrm{SY}(\mathrm{cm})$} & Ön test & 14 & 37,41 & 13,26 & \multirow{2}{*}{0,753} \\
\hline & & & Son test & 14 & 35,16 & 8,98 & \\
\hline & & \multirow{2}{*}{ SG (watt) } & Ön test & 14 & 1575,57 & 513,67 & \multirow{2}{*}{0,396} \\
\hline & & & Son test & 14 & 1495,00 & 417,06 & \\
\hline
\end{tabular}

$*(p<0.05)$, Test: wilcoxon işaretli sıralar testi

Tablo 2' ye bakıldığında; araştırmaya katılan kadın futbolculara uygulanan kor kuvvet antrenmanlarının dikey sıçrama yöntemlerinden; yaylanarak sıçrama (YS) ve Statik sıçrama (SS) değerlerine olumlu düzeyde etki ettiği gözlemlenmiştir $(p<0.05)$. Düsserek sıçrama (DS) değerlerine ise istatistiksel olarak etkisinin olmadığ tespit edilmiştir ( $\mathrm{p}>0.05)$.

\section{Tartışma ve Sonuç}

Kor stabilizasyon, etkili sportif fonksiyonu en üst düzeye çıkaran önemli bir faktördür. Kor stabilizasyon çalışmalarının çoğu kor stabilizasyon ve yaralanmalar arasındaki ilişki üzerine olmakla birlikte, son dönemde kor stabilizasyon ve sportif performans arasındaki ilişkiye dikkat çekilmektedir (Genç, 2018). Kor antrenman programları, kor kasların kuvvetlenmesini ve motor kontrolünü hedefleyen, sporcular ve sedanterler için, hem atletik performansı arttırmada hem de terapik amaçlı kullanılmaktadır (Hibbs ve ark., 2008). 
Göktepe, M., Göktepe, M. M., Güder, F., \& Günay, M. (2019). Kadın futbolculara uygulanan kor kuvvet antrenmanlarının farklı dikey sıçrama yöntemlerine etkisi. Journal of Human Sciences, 16(3), 791-798. doi:10.14687/jhs.v16i3.5637

Kadın futbolculara uygulanan kor kuvvet antrenmanlarının farklı dikey sıçrama yöntemlerine etkisinin belirlenmesi amacıyla yapmış olduğumuz çalışmamızda uygulanan kor kuvvet antrenmanlarının dikey sıçrama yöntemlerinden; yaylanarak sıçrama (YS) ve Statik sıçrama (SS) değerlerine olumlu düzeyde etki ettiği gözlemlenmiştir $(p<0.05)$. Düşerek siçrama (DS) değerlerine ise istatistiksel olarak etkisinin olmadığ tespit edilmiştir ( $\mathrm{p}>0.05)$.

Bir çok araştırmada düzenli uygulatılan kor hareketlerin dikey sıçrama performansını olumlu yönde etkilediği rapor edilmiştir (Doğan ve ark., 2016; Afyon, 2014a; Afyon, 2014b; Butcher ve ark., 2007; Afyon ve Boyac1 2013; Afyon ve Boyac1, 2016; Balaji ve Murugavel, 2013; Sekendiz ve ark., 2010). Yapılan bir çalışmada 19 lise öğrencisi kadın sporcuya haftada 3 gün 7 haftalık uygulanan eğitim programında pliometrik eğitim ile dinamik stabilizasyon ve denge eğitimi karşılaştırılmış, dikey sıçrama testinde her iki grubun da anlamlı bir şekilde sıçrama performansını arttırdığı gözlenmiştir (Myer ve ar., 2006). Başka bir çalışmada Statik, dinamik veya bu iki türün kombinasyonu şeklinde yapılan kor antrenman programları, dikey sıçrama becerisini arttırmıştır (Butcher ve ark., 2007; Cressey ve ark., 2007). 9-12 yaş 17 çocukta $6 * 3$ haftalık kor stabilizasyon programı durarak uzun atlama performansı arttırmıştır (Rahmat ve ark., 2014). Kalaycığlu'nun bale ve modern dans öğrencileri üzerine uygulanan gövde stabilizasyon programı sonucunda non-dominant ve dominant taraf için dikey sıçrama performansı değerlerinde istatistiksel olarak anlamlı artış bulunmuştur (Kalaycıoğlu, 2012). Bu çalışmalar araştırmamızın bulgularından kor kuvvet antrenmanlarının, düşerek dikey sıçramaya etkisi dışında diğer bulgularımızı desteklemektedir. Ayrıca yapılan bir çalışmada stabil olmayan yüzeyde antrenman yapan hem dinamik hem de statik kor çalışma grubunun dikey sıçrama skorları değiștirmemiștir. Antrenman sonucu artan kor stabilizasyon becerisi spor ilişkili performansa etki etmemiştir (Parkhouse ve Ball, 2011). Yapılan diğer çalışmalarda alt ekstremite sıçrama kuvveti, (Steffen ve ark., 2008), yere dik ve yatay düzlemde gerçekleşen reaksiyon kuvveti (Sato ve Mokha, 2009), dikey siçrama (Scibek, 1999) gibi sportif performansı etkileyen faktörler üzerinde anlamlı bir etkisinin olmadığı bildirilmiştir (Gür, 2015). Yapılan bir araştırmada 21 ikinci lig Amerikan futbolcusuna uygulanan çalışmada sporcuların kor güç (sağlık topu yerden mekik ile firlatma ve 60-30 sn maksimum mekik) kor dayanıklilı (McGill protokolü) testleri ile dikey siçrama arasında ilișkiye rastlanılmamıștır (Dendas, 2010). Steffen ve arkadaşları kadın futbolcularda 10 egzersizden oluşan, normal antrenmanının ısınma bölümünde yapılan kor stabilizasyon, denge, dinamik stabilizasyon ve eksantrik hamstring kuvvetini hedef alan çalışmada sonucunda, alt ekstremite izokinetik kuvvetinde, izometrik kalça kuvvetinde, siçrama becerisinde, gelişim görmemişlerdir (Steffen ve ark., 2008). Bu çalışmaların bulguları ise çalışmamızın bulguları ile paralellik göstermemektedir. Fakat araştırmamızda elde ettiğimiz sonuçlardan; kor kuvvet antrenmanlarının düşerek sıçramaya etki etmediği tespit edilmiştir. Bu bağlamda da yukarıda ki araştırmalar çalışmamızı kısmen desteklemektedir. Kadın futbolcularda izometrik kor kuvveti ve fonksiyonel kor kuvvetinin, performans testlerine etkisinin araştırıldığ1 çalışmada, izometrik kor ölçümü gövde fleksiyon ve bilateral rotasyon ölçümleri ile, konsantrik fonksiyonel kor ölçümü, öne abdominal güç ve yana abdominal güç testleri ile yapılmıştır. Performans testi olarak ise topa vuruş hızı ve taç kullanma hızı kullanılmıştır. Bilateral gövde rotasyonu ve bilateral yana abdominal güç testi ile topa vuruş hızı arasında anlamlı bir ilişki varken, izometrik gövde fleksiyonu ve öne abdominal güç testleri ile taç attığı hızı ilişkilidir. Fakat geneline göre izometrik kor kuvveti konsantrik fonksiyonel güç ölçümlerine göre bu iki performans testine daha fazla etki etmiştir. Yazara göre bu ilişki kor stabilizasyonunun, kor kuvvetine göre ekstremite performansına daha fazla etki ettiğini göstermekte ve güç transferinde gövde stabilizasyonunun önemini ortaya koymaktadır (Wagner, 2010; Sever, 2017). Bu araştırma bulguları ise araştırmamızın bulgularını kısmen desteklemektedir.

Sonuç olarak; kadın futbolcularda uygulanan kor kuvvet antrenmanlarının dikey sıçrama yöntemlerinden statik sıçrama ve yaylanarak sıçrama özelliğini geliştirdiği, düşerek sıçrama özelliğine ise etki etmediği söylenebilir. Bu sonuçlar doğrultusunda kadın futbolcularda uygulanacak kor kuvvet antrenmanlarının statik sıçrama ve yaylanarak sıçrama özelliğine olumlu yönde etkisi olabileceğinden antrenman programlarında göz önünde bulundurulması önerilebilir.

\section{Kaynakça}

Afyon, Y.A. (2014a), The Effect of Core and Plyometric Exercises on Soccer Players, International Journal of Contemporary and Applied Studies of Man, Anthropologist, Vol. 18 No.3, p.927-932, India.

Afyon, Y.A. (2014b), Effect of Core training on 16 year-old Soccer Players, Educational Research an Reviews Journals, Vol.9(23), pp 1275-1279. 
Göktepe, M., Göktepe, M. M., Güder, F., \& Günay, M. (2019). Kadın futbolculara uygulanan kor kuvvet antrenmanlarının farklı dikey sıçrama yöntemlerine etkisi. Journal of Human Sciences, 16(3), 791-798. doi:10.14687/jhs.v16i3.5637

Afyon, Y.A., Boyac1, A. (2013), Investigation Of The Effects By Compositely Edited Core-Plyometric Exercises In Sedentary Man OnSome Physical And Motoric Parameters, International Journal of Academic Research,Vol. 5. No. 3. May, 256-261. DOI:10.7813/2075-4124.2013/5-3/A.37Baku, Azerbaija.

Afyon, Y. A., \& Boyac1, A. (2016), The effects of 8-week core training on the development of some motoric features among 18 year-old footballers 18 yaş grubu futbolcularda 8 haftalik merkez bölge (core) antrenmanlarının bazı motorik özelliklerin gelişimine etkisi. Journal of Human Sciences, 13(3), 45954603.

Atan, T., Kabaday1, M., Elioz, M., Cilhoroz, B. T., Akyol, P. (2013), Effect of Jogging and Core Training After Supramaximal Exercise on Recovery. Turkish Journal of Sport and Exercise, 15(1), 73-77.

Balaji, E., Murugavel, K., (2013), Motor fitnes parameters response to core strength training on Handbal Players. International Journal for Life Sciences and Educational Research,1(2):76-80.

Baljinder, S., Ashok, K., ranga, m.D. (2014), Comparison of Vertical Jump Performance of Male Handball \& Basketball Players. Journal of Exercise Science and Physiotherapy, 10(1), 64-68.

Butcher, S.J., Craven, B. R., Chilibeck, P. D., Spink, K. S., Grona, S. L., \& Sprigings, E. J. (2007), The effect of trunk stability training on vertical take off velocity. Journal of Orthopaedic \& Sports Physical Therapy, 37(5), 223-231.

Clark, M.A., (2001), Core stabilization training in rehabilitation. In: Techniques in Musculoskeletal Rehabilitation. Prentice, New York. 259-278.

Cressey, E.M., West, C. A., Tiberio, D. P., Kraemer, W. J., Maresh, C. M. (2007), The effects of ten weeks of lower-body unstable surface training on markers of athletic performance. The Journal of Strength \& Conditioning Research, 21(2), 561-567.

Çon, M., Akyol, P., Tural, E., Taşmektepligil, M. Y. (2012), Voleybolcuların Esneklik ve Vücut Yağ Yüzdesi Değerlerinin, Dikey Stçrama Performansına Etkisi. Selçuk Üniversitesi Beden Eğitimi Ve Spor Bilim Dergisi,14(2), 202-204.

Dendas, A.M., (2010), The relationship between core stability and athletic performance. Doktora Tezi, Humboldt State University.

Dilber, A.O., Lağap, B., Akyüz, Ö., Çoban, C., Akyüz, M., TAŞ, M, \& Özkan, A., (2016). Erkek futbolcularda 8 haftalı kor antrenmanının performansla ilgili fiziksel uygunluk değişkenleri üzerine etkisi. CBÜ Beden Eğitimi ve Spor Bilimleri Dergisi, 11(2), 77-82.

Doğan, G., Mendeş, B., Akcan, F.\& Tepe, A., (2016), The Effects of Eight-Week Core Training on Some Physical and Physiological Parameters of Football Player"Niğde Üniversitesi Beden Eğitimi Ve Spor Bilimleri Dergisi Cilt 10, Say1 1.

Genç, H., (2018), Hentbolcularda Kor Stabilizasyon İle Performans Arasındaki İlişki, Abant İzzet Baysal Üniversitesi Sağlık Bilimleri Enstitüsü, Fizik Tedavi Ve Rehabilitasyon Anabilim Dalı, Yüksek Lisans Tezi.

Günay, M., Yüce, A.İ., (2008), Futbol Antrenmanının Bilimsel Temelleri. Ankara: Gazi Kitabevi.

Günay, M., Tamer, K., Cicioğlu, İ. (2006), Spor Fizyolojisi ve Performans Ölçümü. Ankara: Gazi Kitabevi

Gür, F., (2015), Kor Antrenmanın 8-14 Yaş Grubu Tenis Sporcularının Kor Kuvveti, Statik ve Dinamik Denge Özellikleri Üzerindeki Etkisinin Değerlendirilmesi. Yüksek Lisans Tezi. Ankara Üniversitesi. Sağlık Bilimleri Enstitüsü. Ankara.

Hibbs, A.E., Thompson, K. G., French, D., Wrigley, A. and Spears, I. (2008), Optimizing performance by improving core stability and core strength. Sports Medicine, 38(12), 995-1008.

Kalaycığlu, T., (2012), Bale ve modern dans öğrencilerinde gövde stabilizasyon eğitim programının fiziksel uygunluk üzerine etkisi. Yüksek Lisans Tezi. Hacettepe Üniversitesi. Sağllk Bilimleri Enstitüsü. Ankara.

Moran, G.T., McGlynn, G., (1997), Dynamics of Training and Conditioning. Second Edition. USA: WBC/McGraw-Hill.

Myer, G.D., Ford, K.R., Brent, J.L. and Hewett, T.E., (2006), The effects of plyometric vs. dynamic stabilization and balance training on power, balance, and landing force in female athletes. The Journal of Strength \& Conditioning Research, 20(2), 345-353.

Norris, J.M., Langefeld, C.D., Scherzinger A.L., Rich, S.S., Bookman, E., Beck S.R., Saad, M.F., Haffner, S.M., Bergman, R.N., Bowden D.W. and Wagenknecht, L.E., (2005), Quantitative trait loci for abdominal fat and BMI in hispanic-americans and African-Americans: The IRAS family study, International Journal of Obesity, 29: 67-77 
Göktepe, M., Göktepe, M. M., Güder, F., \& Günay, M. (2019). Kadın futbolculara uygulanan kor kuvvet antrenmanlarının farklı dikey sıçrama yöntemlerine etkisi. Journal of Human Sciences, 16(3), 791-798. doi:10.14687/jhs.v16i3.5637

Parkhouse, K.L., Ball, N., (2011), Influence of dynamic versus static core exercises on performance in field based fitness tests. Journal of bodywork and Movement Therapies, 15(4), 517-524.

Rahmat, A., Naser, H., Belal, M. and Hasan, D., (2014), The effect of core stabilization exercises on the physical fitness in children 9-12 years. Medicina Sportiva: Journal of Romanian Sports Medicine Society, 10(3), 2401.

Reiser, R.F., Rocheford, E.C., Armstrong, C.J., (2006), Building A Better Understanding Of Basic Mechanical Principles Through Analysis Of The Vertical Jump. Strength and Conditioning Journal, 28(4), 70-80.

Riewald, S.T., (2003), Training the "other core". Performance Training Journal,2(3):5-6.

Santana, J.C., (2005), 'Strength training for swimmers: Training the core' Clin J Sport Med,2(27), 40-42.

Sato, K., Mokha, M., (2009), Does core strength training influence running kinetics, lower-extremity stability, and 5000-M performance in runners?. The Journal of Strength \& Conditioning Research, 23 (1), 133 140.

Scibek, J.S., (1999), The effect of core stabilization training on functional performance in swimming.Master Thesis. University of North Carolina at Chapel Hill.

Sekendiz, B., Cug, M., \& Korkusuz, F., (2010), Effects of Swiss-ball core strength training on strength,endurance, flexibility, and balance in sedentary women. The Journal of Strength \& Conditioning Research, 24(11), 3032-3040.

Semler, C.C., (2011), Effect Of Plyometric Training And Squats On The Vertical Jump Performance Of Untrained Females, In Health and Sport Science Dissertation, The University of Memphis.

Sever, O., (2017), Comparison of static and dynamic core exercises' effects on Stork balance test in soccer players. Journal of Human Sciences, 14(2), 1781-1791.

Steffen, K., Bakka, H. M., Myklebust, G., \& Bahr, R., (2008), Performance aspects of an injury prevention program: a ten-week intervention in adolescent female football players. Scandinavian Journal of Medicine \& Science in Sports,18 (5), 596-604.

Tamer, K., (2000), Sporda Fiziksel-Fizyolojik Performansın Ölçülmesi ve Değerlendirilmesi. Ankara: Bağırgan Yayımevi.

Taylor, R.W., Keil, D., Gold, E.J., Williams, S.M., Goulding, A., (1998), Body mass index, waist girth and waistto-hip ratio as indexes of total and regional adiposity in woman: Evalution using receiver operating characteristics curves, The American Journal of Clinical Nutrition, 67:44-49.

Wagner, J. S. (2010), Convergent validity between field tests of isometric core strength, functional core strength, and sport performance variables in female soccer players. Doktora Tezi, Boise State University.

Yildiz, M. (2018). An acute bout of self-myofascial release increases flexibility without a concomitant deficit in muscle performance in football players. International Journal of Physiotherapy, 5(3), 92-97.

\section{Extended English Summary}

In the game of soccer moves like jumping, abrupt halts, short distance sprints, head shot and kicking which are dominated by short timed and dense anaerobic energy system are performed frequently (Günay and Yüce, 2008). The activities containing moves like vertical jumping, jumping and taking off are the ability of firing strength of lower extremity (Günay et al., 2006; Tamer, 2000).

One of the most preferred methods for soccer players is core training (Riewald, 2003). The program aimed for strengthening both pelvic and spine balancing muscles by the performance of the person's own bodyweight is called core training (Atan et al., 2013). Core training is all about regional muscles and their condition (Clark, 2001). Because of the complex structure of performance, it contains unique factors and in the trainings for performance development it has to be well-rounded. The personal success is achieved by the harmonic development of the performance determinative factors (Günay and Yüce, 2008). The vertical jumping is one of the basic skills used in the sports of high competitive power. (Baljinder et al., 2014). The aim in many training models is trying to maximize the ability to jump vertically to increase the performance of the athlete (Baljinder et al., 2014, Reiser et al., 2006). In muscle power and strength based sports there is a crucial need for jumping and promptness (Baljinder et al., 2014, Semler, 2011). 
Göktepe, M., Göktepe, M. M., Güder, F., \& Günay, M. (2019). Kadın futbolculara uygulanan kor kuvvet antrenmanlarının farklı dikey sıçrama yöntemlerine etkisi. Journal of Human Sciences, 16(3), 791-798. doi:10.14687/jhs.v16i3.5637

Recently, there is huge demand for core training and it became an important aspect of training plans (Riewald, 2003). In literature we can see many works of core training having an impact on jumping strength. However among vertical jumping methods there are not many works about its effects on static jumping, counter movement jumping_and drop jumping separately. Also there are less studies on female than men. For this reason we can say that this study has a particular importance. In this context, in this study we aim to state the impact of core strength trainings -applied on female soccer players- to the various vertical jumping methods.

16 volunteer licensed female soccer players of Bartun University Female soccer Team whose age averages are 21,07+-3,56 (year) height averages 163,00+- 7,18 (cm) and bodyweight averages $56,60+-7,15(\mathrm{~kg})$ are involved in the study. Before the tests, volunteer sports female are filled up and signed the questionnaire which shows the state of health and voluntary participation form. These volunteer soccer players are informed with the details of the measurements. The core training program is applied twice a week for 8 weeks regularly. Participators' height are measured by a length gauge (SECA, Germany) sensitive to $0.01 \mathrm{~m}$, and their bodyweights are measured by an electronic weighing machine sensitive to $0.1 \mathrm{~kg}$. (SECA, Germany). (Aydos et al., 2009) . Bmi is measured by dividing bodyweight $\mathrm{kg}$ values to length meter evaluation $(\mathrm{kg} / \mathrm{m} 2)$ (Moran and McGlynn, 1997; Norris et al., 2005; Taylor et al., 1998). The vertical jumping values of participators measured by Powertimer PC 1.9.5 Version Newtest device and the programs of "mat" and computer programs connected to that as divided three types of jumping; static, counter movement and drop jump. All the jumping tests are done three times and they are saved with their measured average values. Jumping height $(\mathrm{cm})(\mathrm{JH})$, and jumping power (watt) (JP) are saved. Static jumping (SJ): Participators, hands on their waists and knees 90 on squat with their two feet on the mat jumped as high as they could. Counter movement jumping(CMJ): The athletes jumped up and down on the mat at the $90^{\circ}$ squat. Drop jumping (DJ): The athletes fell on a mat on the floor with a double foot on a $40 \mathrm{~cm}$ high step, and as soon as their feet touched the mat, they leaped up as much as they could jump with half a squat (Con et al., 2012).

This core training program twice a week for 8 weeks, for 30-45 minutes added to regularly to the end of participators' usual soccer training (Dilber et al., 2016). The training is ended after 10 minutes warm up running by finishing the measurements. This study is held in the BESYO Physiology Laboratory. The statistical evaluation is done by SPSS 22.0 program. The values of the first and last tests are compared statistically by the help of Wilcoxon Marked Lines test after it is checked for normality distribution with Shapiro-Wilk test.

This study, we aimed to determine the effect of core strength training on different vertical jump methods applied to female soccer players. It has been observed that it has a favorable effect on counter movement jumping $(\mathrm{CMJ})$ and static jumping $(\mathrm{SJ})$ values $(\mathrm{p}<0.05)$. It was found that there was no statistically significant effect on drop jumping (DJ) values ( $\mathrm{p}>0.05)$.

As a result; It can be said that the core strength training practiced in female soccer players developed counter movement jumping_and static jumping characteristic of vertical jumping methods and did not affect the drop jumpingfeature. In line with these results, it is recommended to take into account the core training programs because the strength training that will be applied in female soccer players can improve the counter movement jumping and static jumping feature. 